\title{
Effects of yoga, aerobic, and stretching and toning exercises on cognition in adult cancer survivors: protocol of the STAY Fit pilot randomized controlled trial
}

\author{
Neha P. Gothe ${ }^{1 *} \mathbb{D}$, Emily D. Erlenbach ${ }^{1}$, Samuel L. Streeter ${ }^{1}$ and Linda Lehovec ${ }^{2}$
}

\begin{abstract}
Background: Cancer survivors experience compromised quality of life due to impaired cognitive function as a result of cancer diagnosis and treatment. Although exercise has proven to be effective in improving cognitive function across the lifespan, interventions comprehensively testing the effectiveness for cancer survivors are limited. The STAY Fit Trial is a three-armed pilot randomized controlled trial designed to compare the effects of a 12-week yoga, aerobic walking, and stretch and tone intervention on cognitive function among adult cancer survivors.

Methods: This pilot study aims to recruit 75 adult cancer survivors who will complete assessments of cognitive function, cardiovascular fitness, physical activity, and psychosocial measures at baseline and after the 12-week intervention. The aims of STAY Fit are (1) to assess the efficacy of yoga to improve cognitive function among cancer survivors, compared to aerobic exercise and an active control group; (2) to examine changes in cardiovascular fitness as a result of the interventions; and (3) to assess changes in quality of life among our population as a result of the exercise interventions.

Discussion: The STAY Fit Trial will test the effectiveness of yoga, aerobic exercise, and stretching and toning exercises in improving cognitive function and fitness among adult cancer survivors. The results of this pilot study will enable us to understand the most effective physical activity modality to improve cognitive function in this population and potentially combat cancer-related cognitive impairment.
\end{abstract}

Trial registration: ClinicalTrials.gov NCT03650322. Registered on 28 August 2018.

Keywords: Yoga, Cognition, Fitness, Quality of life, Executive function

\section{Background}

Cancer is among the leading causes of death worldwide, yet scientific advances in prevention, diagnosis, and treatment have led to an increased number of cancer survivors estimated to be almost 19 million by 2024 [1]. However, survivorship does not come without an aftermath of physical

\footnotetext{
* Correspondence: npg@illinois.edu

1 Department of Kinesiology and Community Health, University of Illinois at Urbana-Champaign, Urbana, IL 61801, USA

Full list of author information is available at the end of the article
}

health and quality of life complications. In 1998, the President's Cancer Panel and the National Coalition for Cancer Survivorship formally recognized cancer-related cognitive impairment (CRCI) as a quality of life matter that deserved higher priority in clinical research [2]. CRCI is the loss of mental acuity associated with cancer and cancer treatment. About $75 \%$ of all cancer survivors report poorer attention and memory following treatment and the clinical prevalence of treatment-related cognitive impairment ranges from 17 to $75 \%$ [3, 4]. Cognitive processes thought to be

(c) The Author(s). 2020 Open Access This article is licensed under a Creative Commons Attribution 4.0 International License, which permits use, sharing, adaptation, distribution and reproduction in any medium or format, as long as you give appropriate credit to the original author(s) and the source, provide a link to the Creative Commons licence, and indicate if changes were made. The images or other third party material in this article are included in the article's Creative Commons licence, unless indicated otherwise in a credit line to the material. If material is not included in the article's Creative Commons licence and your intended use is not permitted by statutory regulation or exceeds the permitted use, you will need to obtain permission directly from the copyright holder. To view a copy of this licence, visit http://creativecommons.org/licenses/by/4.0/ The Creative Commons Public Domain Dedication waiver (http://creativecommons.org/publicdomain/zero/1.0/) applies to the data made available in this article, unless otherwise stated in a credit line to the data. 
impacted include impairment of memory, learning, concentration, reasoning, executive function, attention, and visual-spatial skills [5]. Despite the prevalence of cognitive impairment in cancer survivors, it is largely underdiagnosed and often left untreated [5, 6].

Although research on the beneficial effects of exercise training on cognitive functioning in non-clinical populations continues to emerge, parallel research examining cognitive functioning in cancer survivors is lagging. Among cancer survivors, many age-related physical and functional declines can be mitigated with exercise training [7]. In intervention studies, regardless of intervention specifics, exercise has been shown to improve quality of life and psychosocial and physical health outcomes in various cancer survivor groups during and after cancer treatment and may also help to manage long-term effects of treatment [8]. A majority of these interventions test aerobic exercise modalities, such as walking, as it is associated with improved health and cognitive function [9, 10]. However, few studies have examined the health benefits of yoga practice on cancer patients [11] and survivors [12].

Yoga is a noteworthy exercise modality that should be examined for its improvements in cognitive function and quality of life in clinical populations. As a holistic, mindbody activity, with components centered on meditation, breathing, and postures, it has shown great potential to improve cognitive function [13, 14]. Moreover, it is a low-impact exercise modality that may be more suitable by people limited in their mobility and function. However, little is known about the potential of yoga in maintaining or enhancing cognitive function and/or reducing CRCI among cancer survivors. An evidence-based review of yoga for cancer patients and survivors [15] reviewed 10 yoga-based interventions and noted some positive results, with outcomes primarily focused on psychosocial health and well-being (quality of life, depression, anxiety and stress being the most common). None of the reviewed interventions addressed cognitive function or CRCI.

STAY Fit is designed as a pilot three-arm randomized controlled trial, comparing the benefits of a 12-week yoga and aerobic walking intervention, using a stretching-toning group as an active control. In line with the purpose of conducting pilot studies, we designed this trial to test the feasibility of our methods and procedures for a future large-scale intervention and to explore possible effects and associations as a function of yoga practice on cognitive health [16]. The primary outcomes are measures of cognitive function, specifically domains of executive function, attention, memory, processing speed, and working memory. Secondary outcomes include cardiovascular fitness changes over the course of the intervention. Tertiary measures will examine changes in selfreported quality of life and psychosocial outcomes. Our protocol has been reported here in accordance with the SPIRIT checklist (see Additional file 1).

\section{Methods \\ Study design}

The STAY Fit trial is a three-arm randomized clinical exercise trial comparing the effects of a 12-week groupbased yoga exercise, aerobic walking, and an active control (stretching and toning) on cognitive function and cardiovascular fitness among adult cancer survivors. We will randomize 75 low active adults between the ages of 30 and 70 years to one of the three conditions. All groups participate in structured group-based exercise led by certified and trained exercise leaders over the course of the 12-week intervention. Study outcomes include cognitive assessments, cardiovascular fitness, and psychosocial questionnaires administered at baseline and following the 12-week intervention.

\section{Duration of study \\ August 2018-May 2020.}

\section{Subjects}

The inclusion criteria are low physical activity engagement (i.e., less than $150 \mathrm{~min}$ of moderate to vigorous exercise per week), including no yoga practice over the last 6 months; aged between 30 and 70 years; capable of performing yoga, aerobic walking, or stretching and toning exercise without any pre-existing condition(s), as determined by their personal physician; the type of cancer (i.e., types of brain cancers are excluded as they could confound with cognitive study outcomes); the last treatment for cancer being at least 1 year from the beginning of the exercise program; not planning to receive any cancer-related treatment during the study period; intention to stay in the Champaign-Urbana area over the duration of the study; comfortable with reading, writing, and speaking English; and willingness to be randomized. Finally, all participants will be required to complete the Physical Activity Readiness Questionnaire (PAR-Q [17];) and must obtain approval from their personal physician, if deemed high-risk on the PAR-Q. The physician approval process will be facilitated by the research staff to minimize participant burden.

\section{Recruitment procedures}

Our recruitment efforts include print, audio, and online media outlets to publicize the STAY Fit study in the Champaign-Urbana communities. All advertising materials include the key characteristics of the study-a free 12-week exercise program, opportunity to meet other cancer survivors from the community, monetary compensation for participation, and free parking. We will advertise in the local newspaper and place short ads on 
local radio, as well as attend various cancer survivorship and awareness events in the local area to distribute flyers and brochures to the attendees. Additionally, we will attend events at the local libraries and churches to distribute brochures. Finally, we will send an email or a letter to participants in the lab database who have been ineligible for previous studies with an invitation to participate in the new STAY Fit study (if deemed eligible). A schedule of enrollment, interventions, and assessments is presented in Fig. 1 .

All participants who express interest will be provided with a description of the study and screened for eligibility. All participants will be required to read and sign a University Institutional Review Board-approved informed consent prior to study participation and data collection. Upon enrollment in the study, each participant will be assigned a random unique identification (ID) number as the only way to match collected data to their identifiable information. An electronic database, stored on a secure passwordprotected network only accessible by the study investigators, will house the key linking participant information and ID number. All written hard copies of participant data collected during the study period will only have the ID number written on them and will be stored in a secure location on the research site. Upon completion of the study, the key linking ID numbers and participant information will be destroyed. Participants will be informed that their de-identified data may be made available to regulatory authorities or ancillary individuals from the university, upon reasonable request. A snapshot of the flow of participants through the STAY Fit study is presented in Fig. 2.

\section{Randomization}

Participants who are eligible for the study and complete all baseline measurements will be randomly assigned to 1 of the 3 intervention arms using a computer-generated program. Randomization will take place in groups following rounds of advertising and is stratified based on sex, age, and years since cancer diagnosis. Couples or friends who sign up for the study together will be randomized together to avoid contamination across groups. Randomization will take place following completion of all baseline assessments and a week before the 12-week exercise sessions are scheduled to begin. Participants will be notified via a mailed letter and follow-up phone calls confirming their randomized group and logistics (parking and directions) for their 12-week exercise sessions.

\begin{tabular}{|c|c|c|c|c|c|}
\hline & \multicolumn{5}{|c|}{ STUDY PERIOD } \\
\hline \multirow[b]{2}{*}{ TIMEPOINT } & Enrollment & Randomization & \multicolumn{2}{|c|}{ Post-allocation } & \multirow{2}{*}{$\frac{\text { Close-out }}{t_{\text {week13 }}}$} \\
\hline & $-t_{\text {week }}$ & 0 & $t_{\text {week } 1}$ & $t_{\text {week } 12}$ & \\
\hline \multicolumn{6}{|l|}{ ENROLLMENT: } \\
\hline Eligibility screen & $\mathrm{X}$ & & & & \\
\hline Informed consent & $\mathrm{X}$ & & & & \\
\hline Randomization & & $X$ & & & \\
\hline \multicolumn{6}{|l|}{ INTERVENTIONS: } \\
\hline \multicolumn{6}{|l|}{ Yoga } \\
\hline \multicolumn{6}{|l|}{ Aerobic walking } \\
\hline \multicolumn{6}{|l|}{ Stretch-n-tone } \\
\hline \multicolumn{6}{|l|}{ ASSESSMENTS: } \\
\hline \multirow{2}{*}{$\begin{array}{r}\text { Cognitive Function } \\
\text { Cardiovascular } \\
\text { fitness }\end{array}$} & $\mathrm{X}$ & & & & $\mathrm{X}$ \\
\hline & $X$ & & & & $\mathrm{X}$ \\
\hline $\begin{array}{l}\text { Well-being and } \\
\text { Quality of Life }\end{array}$ & $\mathrm{X}$ & & & & $\mathrm{X}$ \\
\hline
\end{tabular}

Fig. 1 The STAY Fit Trial schedule of enrollment, interventions, and assessments 
Participant Screening

Pass screening questions and scheduled for orientation and baseline testing session

\begin{tabular}{|c|c|c|c|c|c|}
\hline \multicolumn{6}{|c|}{ Orientation/ First Baseline Testing Session } \\
\hline $\begin{array}{c}\text { Review } \\
\text { and sign } \\
\text { informed } \\
\text { consent }\end{array}$ & \begin{tabular}{|c|} 
Study \\
Orientation
\end{tabular} & $\begin{array}{c}\text { Administer } \\
\text { Mini-Mental } \\
\text { State } \\
\text { Examination }\end{array}$ & $\begin{array}{c}\text { Cognitive } \\
\text { testing }\end{array}$ & $\begin{array}{c}\text { Perform } \\
\text { Submaximal } \\
\text { Treadmill } \\
\text { Test }\end{array}$ & $\begin{array}{c}\text { Receive } \\
\text { questionnaire } \\
\text { and } \\
\text { accelerometer }\end{array}$ \\
\hline
\end{tabular}

\begin{tabular}{|c|c|c|c|}
\hline \multicolumn{4}{|c|}{ Second Baseline Testing Session } \\
\hline $\begin{array}{c}\text { Return } \\
\text { questionnaire and } \\
\text { accelerometer }\end{array}$ & Cognitive testing & $\begin{array}{l}\text { Perform Functional } \\
\text { Fitness test }\end{array}$ & $\begin{array}{l}\text { Receive study } \\
\text { payment }\end{array}$ \\
\hline \multicolumn{4}{|c|}{$\nabla$} \\
\hline \multicolumn{4}{|c|}{ Participant Randomization } \\
\hline \multicolumn{2}{|c|}{$\begin{array}{l}\text { Randomized into one of the three } \\
\text { exercises groups }\end{array}$} & \multicolumn{2}{|c|}{$\begin{array}{l}\text { Mailed information pack with } \\
\text { randomization and information for sessions. }\end{array}$} \\
\hline \\
\hline \multicolumn{4}{|c|}{ STAY Fit Exercise Sessions (12 Weeks) } \\
\hline \multicolumn{2}{|c|}{ Attend exercise sessions } & \multicolumn{2}{|c|}{ Fill out and return weekly home logs } \\
\hline
\end{tabular}

\begin{tabular}{|c|c|c|}
\hline \multicolumn{3}{|c|}{ First Post-Intervention Testing Session } \\
\hline Cognitive testing & $\begin{array}{c}\text { Perform Submaximal } \\
\text { Treadmill Test }\end{array}$ & $\begin{array}{c}\text { Receive questionnaire and } \\
\text { accelerometer }\end{array}$ \\
\hline
\end{tabular}

\begin{tabular}{|c|c|c|c|c|}
\hline \multicolumn{5}{|c|}{ Second Post-Intervention Testing Session } \\
\hline $\begin{array}{c}\text { Return } \\
\text { questionnaire and } \\
\text { accelerometer }\end{array}$ & $\begin{array}{c}\text { Cognitive } \\
\text { testing }\end{array}$ & $\begin{array}{c}\text { Perform } \\
\text { Functional } \\
\text { Fitness test }\end{array}$ & $\begin{array}{c}\text { Receive } \\
\text { study } \\
\text { payment }\end{array}$ & $\begin{array}{c}\text { Complete an } \\
\text { anonymous } \\
\text { program } \\
\text { feedback }\end{array}$ \\
\hline \multicolumn{4}{|r}{ Participant Feedback Report } \\
\hline \multicolumn{4}{|c|}{ Receive thank you letter and program functional fitness report }
\end{tabular}

Fig. 2 Flow of participants through the STAY Fit Trial

This is an open label trial with no blinding of participants or assessors.

\section{Sample size calculation}

We recognize that pilot studies do not typically report formal sample size calculations. However, to aid us in making advertising and recruitment decisions, we ran a very preliminary sample size analysis to get an estimated range of subjects needed. A meta-analysis of yoga effects on cognition [13] indicated an effect size range of $.32-.47$ (Hedge's g) favoring the yoga condition. We based our sample size calculations to adequately power for a small effect within this range. Using an alpha of $0.05 / 2$, a power of 0.80 , and a conservative estimate of a medium effect size $(f=0.35)$, the desired total sample size is 75 ( $N=25$ per group) for this trial.

\section{Measures}

All measures will be assessed at baseline and following the 12-week intervention. Measures of cognitive function (primary outcome) and cardiovascular function (secondary outcome) will be conducted in the laboratory by trained research assistants across two lab visits lasting approximately $2 \mathrm{~h}$ each. A battery of psychosocial questionnaires will be given to the participant to complete at home along with an Actigraph accelerometer (wGT3X-BT) that participants will be asked to wear for 7 days and nights to record objective levels of physical activity and sleep quality. We will schedule the testing appointments 1 week apart, so participants have time to complete the questionnaire packet and wear the accelerometer before returning to the lab. 
Table 1 Battery of neurocognitive tests administered at baseline and follow-up

\begin{tabular}{lll}
\hline Cognitive test & Cognitive domain & Approximate test duration \\
\hline Word list (immediate and delayed recall) & Short- and long-term memory & 5 min \\
Flanker task & Attention and inhibitory control & $8 \mathrm{~min}$ \\
Spatial working memory & Spatial memory & $15 \mathrm{~min}$ \\
Trail making A and B & Visual scanning, mental flexibility, processing speed & $5 \mathrm{~min}$ \\
Matrices & Fluid intelligence & $10 \mathrm{~min}$ \\
Spatial reconstruction task & Relational memory & Self-paced, 20 trials \\
Pattern comparison & Processing speed & 4 min \\
Task switching & Mental flexibility & 15 min \\
\hline
\end{tabular}

\section{Cognitive function (primary outcome)}

The primary outcome of the trial is cognitive function. Specifically, we will measure executive function, attention and processing speed, memory, and working memory using the tests presented in Table 1 . These measures have been used in the exercise-cognition literature and are partly adapted from the NIH Toolbox. The tasks are computer- and paper-based and will be administered over the two testing sessions on separate days and repeated again after the 12-week intervention. The first testing session consists of immediate and delayed word recall [18], flanker task [19], spatial working memory [20, 21], trail making [22], and matrix reasoning [23] task. The second session consists of relational memory [24], pattern comparison [25], and task switching paradigm $[14,21]$. All measures involve a practice phase that explains the task instructions and allows participants to familiarize themselves with the test.

\section{Cardiovascular function (secondary outcome)}

The secondary outcome of the trial is cardiovascular function. A submaximal treadmill test will be administered at baseline and after the 12-week intervention. We will use a modified Balke protocol [26] that estimates a participant's predicted VO2max based on the incline, speed, and heart rate over the last two stages completed during the test. The modified Balke is a well-accepted treadmill protocol that keeps the speed constant $(3.0 \mathrm{mph})$ and workload by increasing the incline by $2.5 \%$ every $3 \mathrm{~min}$. The test will terminate when the participant reaches $85 \%$ of their agepredicted maximum heart rate. We will then use their speed, incline, and heart rate from the last two completed stages, as well as their age-predicted maximum heart rate, to calculate their predicted $\mathrm{VO} 2_{\max }$ [27]. Before the test, anthropometric measurements including height, weight, resting heart rate, and resting blood pressure will be recorded. During the test, blood pressure and a rating of perceived exertion [26] will be recorded every $3 \mathrm{~min}$ for participant safety. Test will conclude once participant's reach their $85 \%$ predicted maximum heart rate. In the event of participants taking prescription medications that are betablockers, a rating of perceived exertion of 17 will serve as an indicator for test termination. All participants will be led into a 3-min walking cool down at $2.0 \mathrm{mph}$ followed by a 2 -min seated rest. Heart rate and blood pressure will be monitored throughout the cool down to ensure participant is back at his/her resting state.

\section{Exercise intervention and active control groups Yoga arm}

The yoga intervention consists of two weekly, 90-min group sessions for 12 weeks. The style of yoga selected for the intervention is Hatha yoga, the most popular

Table 2 Sequence of Hatha yoga and stretching-toning exercises from week 1 of the STAY Fit Trial

\begin{tabular}{|c|c|}
\hline Yoga & Stretching and toning \\
\hline Poses (asanas) & Back arch \\
\hline $\begin{array}{l}\text { Ardha Matsyendrasana-twist in simple } \\
\text { seated pose }\end{array}$ & Forward bend \\
\hline Marjaryasana/Bitilasana — cat and cow & Squat \\
\hline Tadasana-mountain pose & Military press \\
\hline *Utkatasana-chair pose & Walking dips \\
\hline Urdhva Hastasasana-upward salute & Bicep + fly arm cycle \\
\hline *Virabhadrasana-warrior 1 and 2 & Wood chop \\
\hline *Purvottanasana -intense side stretch & Tricep extension \\
\hline $\begin{array}{l}\text { Viparita Karani-Supported legs on the } \\
\text { seat of a chair }\end{array}$ & One leg stand \\
\hline $\begin{array}{l}\text { Supta Baddha Konasana-reclining } \\
\text { bound angle pose }\end{array}$ & Side leg abductions \\
\hline Supported Savasana-corpse pose & Bicycle abdominal twist \\
\hline \multicolumn{2}{|l|}{ Breathing (pranayama) } \\
\hline \multicolumn{2}{|l|}{3 parts breath in Supta Baddha Konasana } \\
\hline \multicolumn{2}{|l|}{ Mediation } \\
\hline $\begin{array}{l}\text { Guided meditation/body scan while in } \\
\text { supported savasana }\end{array}$ & \\
\hline
\end{tabular}

Note: *Using chair for support 
form of yoga practice in the USA [28] which focuses on practicing physical poses, breathing, and meditation. STAY Fit yoga sessions will be held at a local yoga studio and taught by a certified yoga instructor, who has been instructing and practicing for more than 20 years. Each 90 -min session is structured such that participants perform 60-min of stretching and poses (asana), $15 \mathrm{~min}$ of guided breathing practice (pranayama), and $15 \mathrm{~min}$ of guided meditation. Table 2 depicts a sample yoga session. Sessions are designed at a beginner level for the first few weeks, with a focus on helping participants develop good form, proper alignment, and postures for the asanas. Props used during the sessions include a yoga mat, chair, blanket, and blocks.

\section{Aerobic walking intervention arm}

Participants randomized to the walking intervention arm will meet three times a week, 60 min a session, for the 12-week intervention. The intensity of each walking session will be individualized for each participant. The 12-week structure of the walking sessions, including the intensity and duration for each week's sessions, is presented in Table 3 . The rating of perceived exertion (RPE) and target heart rate (HR) zones were selected in accordance with the ACSM exercise prescription guidelines for cancer survivors. Within each 4-week block, participants started at the lowest recommend intensity for $15 \mathrm{~min}$, gradually increasing the duration to $40 \mathrm{~min}$ with the target $\mathrm{HR}$ zone. Prior to the start of the intervention, researchers will calculate each participant's target HR zones using the Kavonen formula [29] ([(HR $\mathrm{Hax}_{\max }-$ $\left.\mathrm{HR}_{\text {rest }}\right) \times \%$ training intensity] $\left.+\mathrm{HR}_{\text {rest }}\right)$, where HRmax $=220-$ age and HRrest $=$ resting heart rate recorded at baseline submaximal treadmill test. All walking sessions are led by trained exercise leaders, who are trained with the detailed protocol for the sessions, and complete 2-h-long orientations to review the manual of procedures and protocols including participant safety, leading warm up and cool down exercises and adapting the intensity of exercise to target the HR zones. During each session, exercise leaders are responsible for monitoring the participant's heart rate every $5 \mathrm{~min}$ to ensure they stay within their designated heart rate zone. Participants

Table 3 ACSM's aerobic exercise prescription for cancer survivors and protocol for the STAY Fit walking group

\begin{tabular}{llll}
\hline Weeks & $\mathbf{1 - 4}$ & $\mathbf{5 - 8}$ & $\mathbf{9 - 1 2}$ \\
\hline Rating of perceived exertion & $12-13$ & $14-15$ & $16-17$ \\
Target heart rate zone (\% of heart rate reserve) & $40-59$ & $60-70$ & $70-89$ \\
Duration (minutes) & $15-40$ & $15-40$ & $15-40$ \\
\hline
\end{tabular}

will be allowed to freely change the speed and/or incline during the session to stay in the target HR zone. If needed, participants will be given wrist and/or ankle weights to wear to keep them at a higher intensity. Sessions will begin with a 5-min warm up including dynamic stretching and brief aerobic activity (e.g., marching in place, high knees) and end with a 5-min slow walking cool down and static stretching until the participant HR returns to pre-exercise resting levels.

\section{Stretching and toning control arm}

Participants randomized into the stretch and toning group will participate in sessions three times a week for 60 min each for the duration of the 12-week program. This group will participate in exercises that target all major muscle groups and work toward balance, toning, and flexibility. A sample stretching and toning exercise sequence is presented in Table 2. A given week will consist of the same exercises, but more reps and/or sets will be added over the course of the week's sessions (Monday to Wednesday to Friday) building from 1 to 2 sets and 8 to 12 reps. Stretches and balance exercises were timed starting at 10 building up to $30 \mathrm{~s}$. Exercises are designed to be progressive and challenging over the 12-week study period. At the beginning of each session, participants will walk around the perimeter of the room for 5 min and then engage in a 5-min warm up stretching routine. During the warm-up and before beginning the exercises, the leaders will inform the participants about the exercises, repetitions, and sets for the session. The exercise leaders will track the number of sets, repetitions, and time for exercises that are time-based (e.g., planks). Exercise leaders will provide posture and breathing cues throughout the session and demonstrate modifications for exercises when a participant presents mobility or functional limitations. The sessions will end with a 5-min cool down consisting of static stretching. Stretching and toning sessions will take place in an exercise studio and will be led by trained exercise leaders. Similar to the walking exercise leaders, these are given a manual detailing the protocol for the sessions and complete 2-h long orientation and training sessions to learn about participant safety, warm-up, stretching and toning exercises and modifications, and cool down stretches.

\section{Exercise logs, attendance, and adherence}

Prior to each session, participants in all groups will be fitted with a chest-strap heart rate monitor (Polar Model F1), which will record their heart rates over the session. At each session, participants will be given an exercise $\log$, where they are to record their resting heart rate, average heart rate for the session, their self-reported 
rating of perceived exertion using the Borg scale [30], and self-reported enjoyment (a 1-5 Likert scale). The supervising exercise leader will keep track of the total minutes of exercise for the day and will review the logs for completeness before concluding the exercise session.

Attendance will be monitored by the respective exercise leaders for the 3 groups. A binder with attendance sheets for the 12 weeks will be presented to the exercise leaders at the first exercise session. Exercise leaders will be responsible for recording attendance and noting announced and unannounced absences. To promote adherence to the study protocols, participants will receive follow-up phone calls for any unannounced absences. They will also be given a mid-session feedback report (6 weeks, i.e., halfway into the intervention), providing averages of their weekly heart rates, rating of perceived exertion, enjoyment ratings, overall attendance percentage, and personalized feedback from their respective exercise leaders. Lastly, two times throughout the study period, exercise leaders will hold a raffle with prizes donated from local businesses (free fitness classes at a local gym) that can be redeemed after the trial ends.

In the event where a participant has decided to drop out of the study, their decision will be noted, and the participant will be contacted to administer follow-up assessments. Participants must have attended at least one session in order to be contacted for follow-up.

\section{Home logs}

Throughout the duration of the 12-week program, the participants will complete weekly home logs to track any participation in physical activity outside of the program. New home logs will be given to participants on Fridays and the completed home logs from the previous week will be collected by the exercise leaders on Mondays. Participants will be encouraged to record the type of physical activity they participated in, the intensity of the activity including an RPE rating and their enjoyment. If the participant does not engage in any home exercise, they will be asked to indicate "None" on the log.

\section{Adverse events and data safety monitoring}

A data safety and monitoring plan has been established and approved by the University Institutional Review Board (IRB) that will allow us to consistently monitor and report adverse events to the University IRB. An official data safety and monitoring committee was not deemed necessary for this trial, as this is a single site, low-risk intervention. However, the investigators and research staff will meet on a weekly basis to review trial proceedings and participant status. All participants will be encouraged to report any health-related changes to the study staff. The exercise leaders will also closely monitor safety and follow up with participants in case of unannounced absences. If a participant discontinues exercise due to a health issue, the research staff will seek permission from their physician for resuming participating in the exercise program. If there is any recurrence of cancer or need for treatment (chemotherapy, radiation, or surgery) during the 12-week exercise sessions for a participant, he/she will be requested by the PI to discontinue participation in the trial as a safety and precautionary measure. Other than this, there will be no special criteria for discontinuing or modifying allocated interventions or stopping the trial.

\section{Statistical analysis}

Data will be analyzed using SPSS 24.0 (IBM Corp., Armonk, NY). Each of the cognitive domains assessed will be analyzed separately. From each of the assessments administered, latent variables will be created to represent a global cognitive score for each domain. These variables will be created for both baseline and post-intervention test scores. Given the pilot nature of this study, we will focus on descriptive statistics and calculating effect sizes (Cohen's d values). Data characteristics permitting (normality, multi-collinearity, sample size), we may conduct additional secondary analyses. For example, a time (baseline, follow up) $\times$ group (yoga, aerobic, stretching-toning) repeated measures analyses of covariance controlling for relevant covariates (possible covariates include age, education, stage of cancer, months since diagnosis, attendance, and baseline performance) can be employed to explore intervention effects for each of the cognitive domains. Similar repeated measures analyses could examine intervention effects for cardiovascular fitness and the tertiary psychosocial outcomes. Given that this is a pilot study, no additional subgroup or adjusted analyses are planned. An intent-totreat analysis approach will be used for any dropouts who completed at least one exercise session. Missing data will be handled using a mean imputation approach. Participants' exercise session attendance and physical activity performed outside of the session (as reported in home logs) will be factored into analyses as appropriate to control for participants' adherence to study protocols.

\section{Discussion}

\section{Strengths, limitations, and future considerations}

To our knowledge, this is the first pilot randomized controlled trial to examine the effects of yoga on cognition in comparison to aerobic exercise and an active control group. Our use of a comprehensive neurocognitive battery will allow us to examine the global and selective effects of exercise on unique cognitive domains. However, this is a 12-week pilot trial and not without limitations. Although a number of yoga and aerobic exercise studies have demonstrated changes in cognitive function 
following 8- to 12-week interventions, longer interventions with multiple measurement time points can provide more evidence pertaining to the "dose-response" effect of exercise. The singular follow-up time point will limit us in assessing the maintenance effects for the study outcomes. Due to limited resources, our secondary outcome involves a submaximal fitness test to estimate cardiovascular fitness changes over the course of the intervention. Future studies could employ long-term follow-ups and a graded maximal exercise test that directly assesses peak $\mathrm{VO}_{2}$ capacity, which is considered the gold standard for cardiovascular measurement. We hope that our pilot study results will aid in informing a fully powered larger efficacy trial in the future. Results from our trial will also allow researchers to utilize more effective measures, perhaps use of magnetic resonance imaging and monitoring physiological data such as blood biomarkers, to investigate possible underlying mechanisms that mediate the exercise-cognition relationship.

\section{Conclusions}

In addition to advancing the science of yoga and contributing to the fields of integrative medicine, aging, neurocognition, and cancer survivorship, the results of this proposed randomized control trial could have a significant impact on health care recommendations for cancer survivorship. Our study will determine the efficacy of yoga in improving cognitive health, and if proven effective, the gentle and amenable nature of yoga practice could have far-reaching applications for cancer survivors, many of whom are aging adults who will experience other age-related disabilities, co-morbidities, and overall compromised physical health. If found comparable to or better than aerobic exercise, yoga practice could be recommended and promoted as an alternative form of physical activity for adult cancer survivors to improve cognition and cancer-related cognitive impairment as well as physical and psychosocial health.

\section{Supplementary information}

Supplementary information accompanies this paper at https://doi.org/10. 1186/s13063-020-04723-2.

Additional file 1. SPIRIT checklist.

\section{Abbreviations}

CRCl: Cancer-related cognitive impairment; PAR-Q: Physical Activity Readiness Questionnaire; RPE: Rating of perceived exertion; HR: Heart rate

\section{Acknowledgements}

We sincerely appreciate our research study participants for their interest and enthusiasm in participating in research and moving the science forward.

\section{Trial status}

ClinicalTrials.gov Identifier NCT03650322, version 1, posted on August 28, 2018 Recruitment began in September 2018; the trial concluded in May 2020.

\section{Protocol amendments}

If a change is to be made to the protocol, the university's Institutional Review Board will be notified and must approve of the changes. Once the approval has been made, the revised protocol will be updated in the

ClinicalTrials.gov registry.

\section{Collection of biological specimens}

This trial does not involve the collection of biological specimens.

\section{Ancillary and post-trial care}

While there is no anticipated harm for trial participation, the sponsoring university and research team does not provide medical or hospitalization insurance coverage for participants in the trial, nor will the university or research team provide compensation for any injury sustained as a result of participation in the trial. There will be no provisions for any post-trial care.

\section{Trial dissemination}

Data obtained throughout the trial period will be used for journal articles, conference presentations, book chapters, and graduate student theses. No publication restrictions have been placed on trial data.

\section{Authors' contributions}

NG: study conception and execution, protocol design, obtained study funding, and manuscript writing. EE and SS: participant recruitment, data collection, manuscript writing. LH: manuscript review and writing. No professional writers have been involved. All named authors adhere to the authorship guidelines of trials and have agreed to publication. The author(s) read and approved the final manuscript.

\section{Funding}

This study is funded by the University of Illinois Campus Research Board Grant RB\#18107. The funder has no role in the study design, collection, analysis and interpretation of data, or preparation of the manuscript.

\section{Availability of data and materials}

This is a study protocol paper, and no datasets were used or analyzed in this manuscript. Requests for more details about the manual of procedures can be directed to the primary corresponding author. Any data required to support the protocol, such as the consent form and other related documentation given to participants, can be supplied on a reasonable request.

\section{Ethics approval and consent to participate}

This study was approved by the University of Illinois at Urbana-Champaign's Institutional Review Board (IRB No. 18922) and is being performed in accordance with the Declaration of Helsinki. All participants read and sign an informed consent prior to participation.

Consent for publication

Not Applicable.

\section{Competing interests}

The authors declare that they have no competing interests.

\section{Author details}

${ }^{1}$ Department of Kinesiology and Community Health, University of Illinois at Urbana-Champaign, Urbana, IL 61801, USA. ²Department of Dance, University of Illinois at Urbana-Champaign, Urbana 61801, USA.

Received: 8 November 2019 Accepted: 3 September 2020

Published online: 15 September 2020

\section{References}

1. Howlader N, Noone A, Krapcho M, Garshell J, Neyman N, Altekruse S, Kosary C, Yu M, Ruhl J, Tatalovich Z. SEER cancer statistics review, 1975-2013. Bethesda: Natl. Cancer Institute; 2013. https://seer.cancer.gov/csr/1975_2 013/.

2. Ahles TA, Saykin A. Cognitive effects of standard-dose chemotherapy in patients with cancer. Cancer Investig. 2001;19:812-20. https://doi.org/10. 1081/CNV-100107743. 
3. Wefel JS, Schagen SB. Chemotherapy-related cognitive dysfunction. Curr Neurol Neurosci Rep. 2012;12:267-75. https://doi.org/10.1007/s11910-0120264-9.

4. Janelsins MC, Kesler SR, Ahles TA, Morrow GR. Prevalence, mechanisms, and management of cancer-related cognitive impairment. Int Rev Psychiatry. 2014;26:102-13.

5. Argyriou AA, Assimakopoulos K, Iconomou G, Giannakopoulou F, Kalofonos HP. Either called "chemobrain" or "chemofog," the long-term chemotherapy-induced cognitive decline in cancer survivors is real. J Pain Symptom Manag. 2011;41:126-39. https://doi.org/10.1016/j.jpainsymman. 2010.04.021.

6. Jean-Pierre P, Johnson-Greene D, Burish TG. Neuropsychological care and rehabilitation of cancer patients with chemobrain: strategies for evaluation and intervention development. Support Care Cancer. 2014;22:2251-60. https://doi.org/10.1007/s00520-014-2162-y.

7. Daum CW, Cochrane SK, Fitzgerald JD, Johnson L, Buford TW. Exercise interventions for preserving physical function among cancer survivors in middle to late life. J Frailty Aging. 2016;5:214-24. https://doi.org/10.14283/ jfa.2016.92.

8. Spence RR, Heesch KC, Brown WJ. Exercise and cancer rehabilitation: a systematic review. Cancer Treat Rev. 2010;36:185-94. https://doi.org/10. 1016/j.ctrv.2009.11.003

9. Smith PJ, Blumenthal JA, Hoffman BM, Cooper H, Strauman TA, WelshBohmer K, Browndyke JN, Sherwood A. Aerobic exercise and neurocognitive performance: a meta-analytic review of randomized controlled trials. Psychosom Med. 2010;72:239

10. Northey JM, Cherbuin N, Pumpa KL, Smee DJ, Rattray B. Exercise interventions for cognitive function in adults older than 50: a systematic review with meta-analysis. Br J Sport Med. 2018;52:154-60.

11. Danhauer SC, Addington EL, Sohl SJ, Chaoul A, Cohen L. Review of yoga therapy during cancer treatment. Support Care Cancer. 2017;25:1357-72. https://doi.org/10.1007/s00520-016-3556-9.

12. Buffart LM, Van Uffelen JGZ, Riphagen II, Brug J, van Mechelen W, Brown WJ, Chinapaw MJM. Physical and psychosocial benefits of yoga in cancer patients and survivors, a systematic review and meta-analysis of randomized controlled trials. BMC Cancer. 2012:12:559.

13. Gothe NP, McAuley E. Yoga and cognition: a meta-analysis of chronic and acute effects. Psychosom Med. 2015;77:784-97. https://doi.org/10.1097/PSY. 0000000000000218

14. Gothe NP, Kramer AF, Mcauley E. The effects of an 8-week hatha yoga intervention on executive function in older adults. J Gerontol A Biol Sci Med Sci. 2014;69. https://doi.org/10.1093/gerona/glu095.

15. Smith KB, Pukall CF. An evidence-based review of yoga as a complementary intervention for patients with cancer. Psychooncology. 2009;18:465-75. https://doi.org/10.1002/pon.1411.

16. Thabane L, Ma J, Chu R, Cheng J, Ismaila A, Rios LP, Robson R, Thabane M, Giangregorio L, Goldsmith $\mathrm{CH}$. A tutorial on pilot studies: the what, why and how. BMC Med Res Methodol. 2010;10:1.

17. Thomas S, Reading J, Shephard RJ. Revision of the Physical Activity Readiness Questionnaire (PAR-Q). Can J Sport Sci. 1992;17(4):338-45. https:// pubmed.ncbi.nlm.nih.gov/1330274/.

18. Salthouse TA. Selective review of cognitive aging. J Int Neuropsychol Soc. 2010;16:754-60.

19. Eriksen BA, Eriksen CW. Effects of noise letters upon the identification of a target letter in a nonsearch task. Percept Psychophys. 1974;16:143-9. https:// doi.org/10.3758/BF03203267

20. Erickson Kl, Prakash RS, Voss MW, Chaddock L, Hu L, Morris KS, White SM, Wojcicki TR, McAuley E, Kramer AF. Aerobic fitness is associated with hippocampal volume in elderly humans. Hippocampus. 2009;19:1030-9. https://doi.org/10.1002/hipo.20547.

21. Voss MW, Prakash RS, Erickson KI, Basak C, Chaddock L, Kim JS, Alves H, Heo S, Szabo AN, White SM, Wójcicki TR, Mailey EL, Gothe N, Olson EA, McAuley E, Kramer AF. Plasticity of brain networks in a randomized intervention trial of exercise training in older adults. Front Aging Neurosci. 2010;2. https://doi. org/10.3389/fnagi.2010.00032

22. Reitan RM. Validity of the trail making test as an indicator of organic brain damage. Percept Mot Skills. 1958;8:271. https://doi.org/10.2466/pms.8.7.271276.

23. Raven JC, John Hugh Court. Raven's progressive matrices and vocabulary scales. Oxford: Oxford pyschologists Press; 1998.
24. Watson PD, Voss JL, Warren DE, Tranel D, Cohen NJ. Spatial reconstruction by patients with hippocampal damage is dominated by relational memory errors. Hippocampus. 2013;23:570-80.

25. Salthouse TA, Babcock RL. Decomposing adult age differences in working memory. Dev Psychol. 1991;27:763-76.

26. Pollock ML, Foster C, Schmidt D, Hellman C, Linnerud AC, Ward A Comparative analysis of physiologic responses to three different maximal graded exercise test protocols in healthy women. Am Heart J. 1982;103: 363-73 http://www.ncbi.nlm.nih.gov/pubmed/7064770.

27. Swain DP, Brawner CA, American College of Sports Medicine. ACSM's resource manual for guidelines for exercise testing and prescription. Philadelphia: Wolters Kluwer Health/Lippincott Williams \& Wilkins; 2014.

28. Clarke TC, Black LI, Stussman BJ, Barnes PM, Nahin RL. Trends in the use of complementary health approaches among adults: United States, 2002-2012. Natl Health Stat Rep. 2015;79:1.

29. Karvonen MJ. The effects of training on heart rate: a longitudinal study. Ann Med Exp Fenn. 1957;35:307-15.

30. Borg GA. Psychophysical bases of perceived exertion. Med Sci Sport Exerc 1982;14. https://doi.org/10.1249/00005768-198205000-00012.

\section{Publisher's Note}

Springer Nature remains neutral with regard to jurisdictional claims in published maps and institutional affiliations.
Ready to submit your research? Choose BMC and benefit from:

- fast, convenient online submission

- thorough peer review by experienced researchers in your field

- rapid publication on acceptance

- support for research data, including large and complex data types

- gold Open Access which fosters wider collaboration and increased citations

- maximum visibility for your research: over $100 \mathrm{M}$ website views per year

At BMC, research is always in progress.

Learn more biomedcentral.com/submissions 